\title{
Learning dyadic data and predicting unaccomplished co- occurrent values by mixture model
}

\author{
Loc Nguyen \\ Independent scholar, Vietnam \\ Email: ng_phloc@yahoo.com \\ Homepage: www.locnguyen.net
}

\begin{abstract}
Dyadic data which is also called co-occurrence data (COD) contains co-occurrences of objects. Searching for statistical models to represent dyadic data is necessary. Fortunately, finite mixture model is a solid statistical model to learn and make inference on dyadic data because mixture model is built smoothly and reliably by expectation maximization (EM) algorithm which is suitable to inherent spareness of dyadic data. This research summarizes mixture models for dyadic data. When each co-occurrence in dyadic data is associated with a value, there are many unaccomplished values because a lot of co-occurrences are inexistent. In this research, these unaccomplished values are estimated as mean (expectation) of random variable given partial probabilistic distributions inside dyadic mixture model.
\end{abstract}

Keywords: dyadic data, co-occurrence data, expectation maximization (EM) algorithm, mixture model.

\section{Introduction}

Suppose data has two parts such as hidden part $X$ and observed part $Y$ and we only know $Y$. A relationship between random variable $X$ and random variable $Y$ is specified by the joint probabilistic density function (PDF) denoted $f(X, Y \mid \Theta)$ where $\Theta$ is parameter. Given sample $\mathcal{Y}=\left\{Y_{1}, Y_{2}, \ldots, Y_{N}\right\}$ whose all $Y_{i}$ (s) are mutually independent and identically distributed (iid), it is required to estimate $\Theta$ based on $\mathcal{Y}$ whereas $X$ is unknown. Expectation maximization (EM) algorithm is applied to solve this problem when only $\mathcal{Y}$ is observed. EM has many iterations and each iteration has two steps such as expectation step (E-step) and maximization step (Mstep). At some $t^{\text {th }}$ iteration, given current parameter $\Theta^{(t)}$, the two steps are described as follows: E-step:

The expectation $Q\left(\Theta \mid \Theta^{(t)}\right)$ is determined based on current parameter $\Theta^{(t)}$, according to equation 1.1 (Nguyen, 2020, p. 50).

$$
Q\left(\Theta \mid \Theta^{(t)}\right)=\sum_{i=1}^{N} \int_{X} f\left(X \mid Y_{i}, \Theta\right) \log \left(f\left(X, Y_{i} \mid \Theta^{\prime}\right)\right) \mathrm{d} X
$$

M-step:

The next parameter $\Theta^{(t+1)}$ is a maximizer of $Q\left(\Theta \mid \Theta^{(t)}\right)$ with subject to $\Theta$. Note that $\Theta^{(t+1)}$ will become current parameter at the next iteration (the $(t+1)^{\text {th }}$ iteration).

Table 1.1. E-step and M-step of EM algorithm

EM algorithm will converge after some iterations, at that time we have the estimate $\Theta^{(t)}=\Theta^{(t+1)}$ $=\Theta^{*}$. Note, the estimate $\Theta^{*}$ is result of EM. The EM algorithm shown in table 1.1 is also called general EM or GEM.

Especially, the random variable $X$ represents latent class or latent component of random variable $Y$. Suppose $X$ is discrete and ranges in $\{1,2, \ldots, K\}$. As a convention, let $k=X$. Note, because all $Y_{i}(\mathrm{~s})$ are iid, let random variable $Y$ represent every $Y_{i}$. The so-called probabilistic finite mixture model is represented by the PDF of $Y$, as follows: 
Where,

$$
f(Y \mid \Theta)=\sum_{k=1}^{K} \alpha_{k} f_{k}\left(Y \mid \theta_{k}\right)
$$

$$
\begin{aligned}
& \Theta=\left(\alpha_{1}, \alpha_{2}, \ldots, \alpha_{K}, \theta_{1}, \theta_{2}, \ldots, \theta_{K}\right)^{T} \\
& \sum_{k=1}^{K} \alpha_{k}=1
\end{aligned}
$$

Note, the superscript " $T$ " denotes transpose operator for vector and matrix. The $Q\left(\Theta \mid \Theta^{(t)}\right)$ is re-defined for finite mixture model as follows (Nguyen, 2020, p. 79):

$$
Q\left(\Theta \mid \Theta^{(t)}\right)=\sum_{i=1}^{N} \sum_{k=1}^{K} P\left(k \mid Y_{i}, \Theta^{(t)}\right) \log \left(\alpha_{k} f_{k}\left(Y_{i} \mid \theta_{k}\right)\right)
$$

Where,

$$
P\left(k \mid Y_{i}, \Theta^{(t)}\right)=\frac{\alpha_{k}^{(t)} f_{k}\left(Y_{i} \mid \theta_{k}^{(t)}\right)}{\sum_{l=1}^{K} \alpha_{l}^{(t)} f_{l}\left(Y_{i} \mid \theta_{l}^{(t)}\right)}
$$

An interesting application of finite mixture model is soft clustering. Traditional clustering methods assign a fixed cluster to every data point in sample, which means that every data point belongs exactly to one cluster. Soft clustering is more flexible when every data point belongs to more than one cluster and the degree of assignment is represented by a probability. It is easy to recognize that when mixture model is applied into soft clustering, latent class $k$ represents a cluster.

Every observation in ordinary sample is univariate or multivariate but there is a case that ordinary sample becomes dyadic sample related to two sets of objects, which causes some modifications of mixture model. Dyadic data which is also called co-occurrence data (COD) contains co-occurrent events of objects. It is necessary to obtain statistical models to represent dyadic data and fortunately, finite mixture model is the one. Recall that EM is applied to learn mixture model. The next section focuses on mixture model for dyadic data.

\section{Mixture models for dyadic data}

Given two finite sets $\mathcal{X}=\left\{x_{1}, x_{2}, \ldots, x_{N}\right)$ and $\mathcal{Y}=\left\{y_{1}, y_{2}, \ldots, y_{M}\right)$ with note that $x_{i}(\mathrm{~s})$ and $y_{j}(\mathrm{~s})$ represent $\mathcal{X}$-objects and $\mathcal{Y}$-objects, respectively; exactly, they are names of objects. The numbers of $\mathcal{X}$-objects and $\mathcal{Y}$-objects are $|X|=N$ and $|\mathcal{Y}|=M$, respectively. For example, in information retrieval, $x_{i}(\mathrm{~s})$ are documents and $y_{j}(\mathrm{~s})$ are keywords. Hence, $x_{i}$ and $y_{j}$ are not evaluated as numbers. An observational pair $\left(x_{i}, y_{j}\right) \in \mathcal{X} \times \mathcal{Y}$ is called a co-occurrence of $x_{i}$ and $y_{j}$. Dyadic data or COD $\mathcal{S}$ contains these co-occurrences with note that a co-occurrence $\left(x_{i}\right.$, $\left.y_{j}\right)$ can exist more than one time. So, each co-occurrence $\left(x_{i}, y_{j}\right)$ is indexed by an index $r$. As a result, each co-occurrence is denoted by the triple $\left(x_{i}, y_{j}, r\right)$ and we have (Hofmann \& Puzicha, 1998, p. 1):

Where,

$$
\mathcal{S}=\left\{\left(x_{i}, y_{j}, r\right): 1 \leq r \leq|\mathcal{S}|\right\}
$$

$$
\begin{aligned}
& x_{i} \in \mathcal{X}=\left\{x_{1}, x_{2}, \ldots, x_{|x|}\right\} \\
& y_{j} \in \mathcal{Y}=\left\{y_{1}, y_{2}, \ldots, y_{|y|}\right\}
\end{aligned}
$$

Of course, the size of $\mathcal{S}$ is $|\mathcal{S}|$. As a convention, $x_{i}(r)$ and $y_{j}(r)$ indicate that $\mathcal{X}$-object and $\mathcal{Y}$ object at the $r^{\text {th }}$ co-occurrence are $x_{i}$ and $y_{j}$, respectively. Thus, the triplet $\left(x_{i}, y_{j}, r\right)$ can be denoted as $\left(x_{i}(r), y_{j}(r), r\right)$. For example, suppose $\mathcal{X}=\left\{x_{1}, x_{2}, x_{3}\right)$ and $\mathcal{Y}=\left\{y_{1}, y_{2}\right)$, and dyadic data of 4 co-occurrences, $\mathcal{S}=\left\{\left(x_{1}, y_{1}, 1\right),\left(x_{1}, y_{1}, 2\right),\left(x_{1}, y_{2}, 3\right),\left(x_{1}, y_{1}, 4\right)\right\}$, we observe that $x_{1}$ and $y_{1}$ occur together three times at $r=1, r=2$, and $r=4$ where as $x_{1}$ and $y_{2}$ occur together one 
time at $r=3$. In the first co-occurrence $\left(x_{1}, y_{1}, 1\right)$, the notation $x_{1}(1)$ indicate that the $\mathcal{X}$-object at this co-occurrence is $x_{1}$. In the third co-occurrence $\left(x_{1}, y_{2}, 3\right)$, the notation $y_{2}(3)$ indicate that the $\mathcal{Y}$-object at this co-occurrence is $y_{2}$.

If each co-occurrence of $x_{i}$ and $y_{j}$ is associated with a value $z$ (Hofmann, Puzicha, \& Jordan, Learning from Dyadic Data, 1998, p. 1), the triple $\left(x_{i}, y_{j}, r\right)$ becomes the quadruplet $\left(x_{i}, y_{j}, z, r\right)$ which is called valued co-occurrence of $x_{i}$ and $y_{j}$. The value $z$ is called associative value or cooccurrent value. If $z$ is value of a variable $Z$ then, $Z$ is called associative variable or co-occurrent variable. As a result, the sample $\mathcal{S}$ is called valued dyadic data or valued COD. Note, $Z$ can be univariate or multivariate (vector).

Where,

$$
\mathcal{S}=\left\{\left(x_{i}, y_{j}, Z, r\right): 1 \leq r \leq|\mathcal{S}|\right\}
$$

$$
\begin{aligned}
& x_{i} \in \mathcal{X}=\left\{x_{1}, x_{2}, \ldots, x_{|x|}\right\} \\
& y_{j} \in \mathcal{Y}=\left\{y_{1}, y_{2}, \ldots, y_{|y|}\right\}
\end{aligned}
$$

As a convention, $Z(r)$ or $z(r)$ indicates that the associative value at $r^{\text {th }}$ co-occurrence is $Z=z$. Thus, the quadruplet $\left(x_{i}, y_{j}, Z, r\right)$ can be denoted as $\left(x_{i}(r), y_{j}(r), Z(r), r\right)$. For example, suppose $\mathcal{X}=\left\{x_{1}, x_{2}, x_{3}\right)$ and $\mathcal{Y}=\left\{y_{1}, y_{2}\right)$, and dyadic sample of 4 co-occurrences, $\mathcal{S}=\left\{\left(x_{1}, y_{1}, 6,1\right),\left(x_{1}\right.\right.$, $\left.\left.y_{1}, 8,2\right),\left(x_{1}, y_{2}, 7,3\right),\left(x_{1}, y_{1}, 9,4\right)\right\}$, we observe that $x_{1}$ and $y_{1}$ occur together three times at $r=1$, $r=2$, and $r=4$ where as $x_{1}$ and $y_{2}$ occur together one time at $r=3$. Moreover, at $r=1, r=2, r=3$, and $r=4$, associative values are $Z(1)=6, Z(2)=7, Z(3)=8$, and $Z(4)=9$, respectively. Valued dyadic data is special case of dyadic data. As a convention, dyadic data is default if there is no additional information.

Given fixed $x_{k}$, let $\mathcal{S}_{x_{k}}$ be the $\mathcal{X}$-partitioned subset of $\mathcal{S}$ which contains co-occurrences whose $\mathcal{X}$-objects are fixed at $x_{k}$ (Hofmann \& Puzicha, Statistical Models for Co-occurrence Data, 1998, p. 1). Note, $\mathcal{S}_{x_{k}}$ can be empty. The size of $\mathcal{S}_{x_{k}}$ is $\left|\mathcal{S}_{x_{k}}\right|$.

$$
\delta_{x_{k}}=\left\{\left(x_{i}, y_{j}, z, r\right): x_{i}=x_{k}\right\}
$$

Dyadic data $\mathcal{S}$ is partitioned into $|X|$ subsets $\mathcal{S}_{x_{k}}$.

$$
\begin{aligned}
& \delta=\bigcup_{k=1}^{|X|} \delta_{x_{k}} \\
& \forall i \neq j, \mathcal{S}_{x_{i}} \cap \mathcal{S}_{x_{j}}=\varnothing
\end{aligned}
$$

Given fixed $y_{l}$, let $\delta_{y_{l}}$ be the $\mathcal{Y}$-partitioned subset of $\mathcal{S}$ which contains co-occurrences whose $\mathcal{Y}$-objects are fixed at $y_{l}$. Note, $\delta_{y_{l}}$ can be empty. The size of $\delta_{y_{l}}$ is $\left|\mathcal{S}_{y_{l}}\right|$.

$$
\delta_{y_{l}}=\left\{\left(x_{i}, y_{j}, z, r\right): y_{j}=y_{l}\right\}
$$

Dyadic data $\mathcal{S}$ is partitioned into $|\mathcal{Y}|$ subsets $\mathcal{S}_{y_{l}}$.

$$
\begin{aligned}
& \mathcal{S}=\bigcup_{\substack{l=1 \\
\forall i \neq j,}}^{|y|} \mathcal{S}_{y_{l}} \cap \mathcal{S}_{y_{j}}=\varnothing \\
& \forall
\end{aligned}
$$

Given fixed $x_{k}$ and fixed $y_{l}$, let $\mathcal{S}_{x_{k} y_{l}}$ be the subset of the $\mathcal{S}$ which contains co-occurrences whose $\mathcal{X}$-objects and $\mathcal{Y}$-objects are fixed at $x_{k}$ and $y_{l}$. Note, $\delta_{x_{k} y_{l}}$ can be empty. The size of $\mathcal{S}_{x_{k} y_{l}}$ is $\left|\mathcal{S}_{x_{k} y_{l}}\right|$.

$$
\delta_{x_{k} y_{l}}=\left\{\left(x_{i}, y_{j}, z, r\right): x_{i}=x_{k}, y_{j}=y_{l}\right\}
$$

Let $n\left(x_{i}\right)$ and $n\left(y_{j}\right)$ denote the number of $x_{i}$ and the number of $y_{j}$, respectively.

$$
\begin{aligned}
& n\left(x_{i}\right)=\left|\mathcal{S}_{x_{i}}\right| \\
& n\left(y_{j}\right)=\left|\mathcal{S}_{y_{j}}\right|
\end{aligned}
$$


Let $n\left(x_{i}, y_{j}\right)$ denote the number of co-occurrences $\left(x_{i}, y_{j}\right)$.

$$
n\left(x_{i}, y_{j}\right)=\left|\mathcal{S}_{x_{i} y_{j}}\right|
$$

Let $n\left(x_{i} \mid y_{j}\right)$ and $n\left(y_{j} \mid x_{i}\right)$ denote the frequency of $x_{i}$ given $y_{j}$ and the frequency of $y_{j}$ given $x_{i}$, respectively.

$$
\begin{aligned}
& n\left(x_{i} \mid y_{j}\right)=\frac{n\left(x_{i}, y_{j}\right)}{n\left(y_{j}\right)} \\
& n\left(y_{j} \mid x_{i}\right)=\frac{n\left(x_{i}, y_{j}\right)}{n\left(x_{i}\right)}
\end{aligned}
$$

For example, suppose $\mathcal{X}=\left\{x_{1}, x_{2}, x_{3}\right)$ and $\mathcal{Y}=\left\{y_{1}, y_{2}\right)$, and dyadic data of 4 co-occurrences, $\mathcal{S}$ $=\left\{\left(x_{1}, y_{1}, 1\right),\left(x_{1}, y_{1}, 2\right),\left(x_{1}, y_{2}, 3\right),\left(x_{1}, y_{1}, 4\right)\right\}$, we have $\delta_{x_{1}}=\left\{\left(x_{1}, y_{1}, 1\right),\left(x_{1}, y_{1}, 2\right),\left(x_{1}, y_{2}, 3\right)\right.$, $\left.\left(x_{1}, y_{1}, 4\right)\right\}, \mathcal{S}_{x_{2}}=\mathcal{S}_{x_{3}}=\emptyset, \mathcal{S}_{y_{1}}=\left\{\left(x_{1}, y_{1}, 1\right),\left(x_{1}, y_{1}, 2\right),\left(x_{1}, y_{1}, 4\right)\right\}, \mathcal{S}_{y_{2}}=\left\{\left(x_{1}, y_{2}, 3\right)\right\}, \mathcal{S}_{x_{1} y_{1}}=$ $=\left\{\left(x_{1}, y_{1}, 1\right),\left(x_{1}, y_{1}, 2\right),\left(x_{1}, y_{1}, 4\right)\right\}, \mathcal{\delta}_{x_{1} y_{2}}=\left\{\left(x_{1}, y_{2}, 3\right)\right\}, \delta_{x_{2} y_{1}}=\mathcal{S}_{x_{2} y_{2}}=\mathcal{S}_{x_{3} y_{1}}=\mathcal{S}_{x_{3} y_{2}}=\emptyset$, $n\left(x_{1}\right)=1, n\left(x_{2}\right)=n\left(x_{3}\right)=0, n\left(y_{1}\right)=3, n\left(y_{2}\right)=1, n\left(x_{1}, y_{1}\right)=3, n\left(x_{1}, y_{2}\right)=1, n\left(x_{2}, y_{1}\right)=n\left(x_{2}, y_{2}\right)$ $=n\left(x_{3}, y_{1}\right)=n\left(x_{3}, y_{2}\right)=0, n\left(x_{1} \mid y_{1}\right)=1, n\left(x_{1} \mid y_{2}\right)=1, n\left(x_{2} \mid y_{1}\right)=n\left(x_{2} \mid y_{2}\right)=n\left(x_{3} \mid y_{1}\right)=n\left(x_{3} \mid\right.$ $\left.y_{2}\right)=0, n\left(y_{1} \mid x_{1}\right)=3 / 4, n\left(y_{2} \mid x_{1}\right)=1 / 4$.

Suppose each co-occurrence $\left(x_{i}, y_{j}\right)$ belongs to a latent variable $C$ and $C$ has $K$ values $c_{k}(\mathrm{~s})$. These values $c_{k}(\mathrm{~s})$ are called classes or aspects and thus, mixture model for dyadic data is also called aspect model or latent class model which aims to discover the latent variable $C$. Without loss of generality, let $c_{k}=k$ where $k=1,2, \ldots, K$. The random variable $C$ has discrete distribution such that every value has an associated probability $\alpha_{k}$. Of course, there are $K$ probabilities $\alpha_{k}(\mathrm{~s})$. There are three kinds of dyadic mixture model for dyadic data such as symmetric mixture model (SMM), asymmetric mixture model (AMM), and product-space mixture model (PMM). This section only explains these models when they were introduced by Hofmann and Puzicha (Hofmann \& Puzicha, Statistical Models for Co-occurrence Data, 1998).

The mixture model of dyadic data is called symmetric mixture model (SMM) if $\alpha_{k}$ (s) are independent from both $x_{i}$ and $y_{j}$. SMM is defined as follows (Hofmann \& Puzicha, Statistical Models for Co-occurrence Data, 1998, p. 2):

$$
P\left(x_{i}, y_{j} \mid \Theta\right)=\sum_{k=1}^{K} \alpha_{k} P\left(x_{i}, y_{j} \mid k\right)=\sum_{k=1}^{K} \alpha_{k} p_{i \mid k} q_{j \mid k}
$$

Where $\alpha_{k}$ is the probability of aspect $k$. Note, $P($.) denote probability.

The $p_{i \mid k}$ is the probability of $x_{i}$ given aspect $k$.

$$
\alpha_{k}=P(k)
$$

The $q_{j \mid k}$ is the probability of $y_{j}$ given aspect $k$.

$$
p_{i \mid k}=P\left(x_{i} \mid k\right)
$$

$$
q_{j \mid k}=P\left(y_{j} \mid k\right)
$$

This implies that $x_{i}$ and $y_{j}$ are mutually independent in SMM.

$$
P\left(x_{i}, y_{j} \mid k\right)=P\left(x_{i} \mid k\right) P\left(y_{j} \mid k\right)
$$

The joint probability of $x_{i}, y_{j}$, and $k$ is:

$$
P\left(x_{i}, y_{j}, k\right)=P(k) P\left(x_{i}, y_{j} \mid k\right)=\alpha_{k} P\left(x_{i} \mid k\right) P\left(y_{j} \mid k\right)=\alpha_{k} p_{i \mid k} q_{j \mid k}
$$

The parameter of SMM is $\Theta=\left(\alpha_{k}, p_{i \mid k}, q_{j k k}\right)^{T}$ in which there are $K(|X|+|\mathcal{Y}|+1)$ partial parameters $\alpha_{k}, p_{i \mid k}$, and $q_{j \mid k}$. Note,

$$
\sum_{k=1}^{K} \alpha_{k}=1, \sum_{i=1}^{|x|} p_{i \mid k}=1, \sum_{j=1}^{|y|} q_{j \mid k}=1
$$

By applying GEM, given dyadic sample $\mathcal{S}$, at the $t^{\text {th }}$ iteration of GEM, given current parameter $\Theta^{(t)}=\left(\alpha_{k}^{(t)}, p_{i \mid k^{(t)}}, q_{j \mid k}{ }^{(t)}\right)^{T}$, the conditional expectation $Q\left(\Theta \mid \Theta^{(t)}\right)$ is: 
Where,

$$
\begin{aligned}
Q\left(\Theta \mid \Theta^{(t)}\right)= & \sum_{r=1}^{|\mathcal{S}|} \sum_{k=1}^{K} P\left(k \mid x_{i}(r), y_{j}(r), \Theta^{(t)}\right) \log \left(\alpha_{k} p_{i \mid k} q_{j \mid k}\right) \\
& =\sum_{i=1}^{|X|} \sum_{j=1}^{|y|} n\left(x_{i}, y_{j}\right) \sum_{k=1}^{K} P\left(k \mid x_{i}, y_{j}, \Theta^{(t)}\right)\left(\log \left(\alpha_{k}\right)+\log \left(p_{i \mid k}\right)\right. \\
& \left.+\log \left(q_{j \mid k}\right)\right)
\end{aligned}
$$

$$
P\left(k \mid x_{i}, y_{j}, \Theta^{(t)}\right)=\frac{\alpha_{k}^{(t)} p_{i \mid k}^{(t)} q_{j \mid k}^{(t)}}{\sum_{l=1}^{K} \alpha_{l}^{(t)} p_{i \mid l}^{(t)} q_{j \mid l}^{(t)}}
$$

Note, $n\left(x_{i}, y_{j}\right)$ is the number of co-occurrences $\left(x_{i}, y_{j}\right)$ in $\mathcal{S}$, which is specified by equation 2.7 . Please refer to equation 1.4 to comprehend equation 2.11. Because there are three constraints

$$
\sum_{k=1}^{K} \alpha_{k}=1, \sum_{i=1}^{|x|} p_{i \mid k}=1, \sum_{j=1}^{|y|} q_{j \mid k}=1
$$

We use Lagrange duality method to maximize to maximize $Q\left(\Theta \mid \Theta^{(t)}\right)$. The Lagrange function $l a\left(\Theta, \lambda \mid \Theta^{(t)}\right)$ is sum of $Q\left(\Theta \mid \Theta^{(t)}\right)$ and these constraints, as follows:

$$
\begin{aligned}
l a\left(\Theta, \lambda \mid \Theta^{(t)}\right)= & Q\left(\Theta \mid \Theta^{(t)}\right)+\lambda_{1}\left(1-\sum_{k=1}^{K} \alpha_{k}\right)+\lambda_{2}\left(1-\sum_{i=1}^{|x|} p_{i \mid k}\right)+\lambda_{3}\left(1-\sum_{j=1}^{|y|} q_{j \mid k}\right) \\
& =\sum_{i=1}^{|x|} \sum_{j=1}^{|y|} n\left(x_{i}, y_{j}\right) \sum_{k=1}^{K} P\left(k \mid x_{i}, y_{j}, \Theta^{(t)}\right)\left(\log \left(\alpha_{k}\right)+\log \left(p_{i \mid k}\right)+\log \left(q_{j \mid k}\right)\right) \\
& +\lambda_{1}\left(1-\sum_{k=1}^{K} \alpha_{k}\right)+\lambda_{2}\left(1-\sum_{i=1}^{|X|} p_{i \mid k}\right)+\lambda_{3}\left(1-\sum_{j=1}^{|y|} q_{j \mid k}\right)
\end{aligned}
$$

Note, $\lambda=\left(\lambda_{1}, \lambda_{2}, \lambda_{3}\right)^{T}$ where $\lambda_{1} \geq 0, \lambda_{2} \geq 0$, and $\lambda_{3} \geq 0$ are called Lagrange multipliers. Of course, $l a\left(\Theta, \lambda \mid \Theta^{(t)}\right)$ is function of $\Theta$ and $\lambda$. The next parameters $\Theta^{(t+1)}$ that maximizes $Q\left(\Theta \mid \Theta^{(t)}\right)$ at $\mathrm{M}$ step of some $t^{\text {th }}$ iteration is solution of the equation formed by setting the first-order partial derivatives of Lagrange function regarding $\Theta$ and $\lambda$ to be zero.

The first-order partial derivative of Lagrange function regarding $\alpha_{k}$ is:

$$
\frac{\partial l a\left(\Theta, \lambda \mid \Theta^{(t)}\right)}{\partial \alpha_{k}}=\sum_{i=1}^{|x|} \sum_{j=1}^{|y|} n\left(x_{i}, y_{j}\right) \frac{1}{\alpha_{k}} P\left(k \mid x_{i}, y_{j}, \Theta^{(t)}\right)-\lambda_{1}
$$

Setting this partial derivative to be zero, we obtain:

$$
\sum_{i=1}^{|x|} \sum_{j=1}^{|y|} n\left(x_{i}, y_{j}\right) P\left(k \mid x_{i}, y_{j}, \Theta^{(t)}\right)-\alpha_{k} \lambda_{1}=0
$$

Summing the equation above over $K$ aspects $\{1,2, \ldots, K\}$, we have:

$$
\begin{aligned}
& \sum_{i=1}^{|X|} \sum_{j=1}^{|y|} n\left(x_{i}, y_{j}\right) \sum_{k=1}^{K} P\left(k \mid x_{i}, y_{j}, \Theta^{(t)}\right)-\lambda_{1} \sum_{k=1}^{K} \alpha_{k}=0 \\
& \Leftrightarrow \sum_{i=1}^{|X|} \sum_{j=1}^{|Y|} n\left(x_{i}, y_{j}\right)-\lambda_{1}=0 \Leftrightarrow \lambda_{1}=\sum_{i=1}^{|X|} \sum_{j=1}^{|y|} n\left(x_{i}, y_{j}\right)
\end{aligned}
$$

This means the next parameters $\alpha_{k}{ }^{(t+1)}$ is: 


$$
\alpha_{k}^{(t+1)}=\frac{\sum_{i=1}^{|X|} \sum_{j=1}^{|y|} n\left(x_{i}, y_{j}\right) P\left(k \mid x_{i}, y_{j}, \Theta^{(t)}\right)}{\sum_{i=1}^{|X|} \sum_{j=1}^{|y|} n\left(x_{i}, y_{j}\right)}
$$

The first-order partial derivative of Lagrange function regarding $p_{i \mid k}$ is:

$$
\frac{\partial l a\left(\Theta, \lambda \mid \Theta^{(t)}\right)}{\partial p_{i \mid k}}=\sum_{j=1}^{|y|} n\left(x_{i}, y_{j}\right) \frac{1}{p_{i \mid k}} P\left(k \mid x_{i}, y_{j}, \Theta^{(t)}\right)-\lambda_{2}
$$

Setting this partial derivative to be zero, we obtain:

$$
\sum_{j=1}^{|y|} n\left(x_{i}, y_{j}\right) P\left(k \mid x_{i}, y_{j}, \Theta^{(t)}\right)-p_{i \mid k} \lambda_{2}=0
$$

Summing the equation above over $\mathcal{X}$, we have:

$$
\begin{aligned}
& \sum_{i=1}^{|X|} \sum_{j=1}^{|y|} n\left(x_{i}, y_{j}\right) P\left(k \mid x_{i}, y_{j}, \Theta^{(t)}\right)-\lambda_{2} \sum_{i=1}^{|X|} p_{i \mid k}=0 \\
& \Leftrightarrow \lambda_{2}=\sum_{i=1}^{|X|} \sum_{j=1}^{|y|} n\left(x_{i}, y_{j}\right) P\left(k \mid x_{i}, y_{j}, \Theta^{(t)}\right)
\end{aligned}
$$

This means the next parameters $p_{i \mid k}{ }^{(t+1)}$ is:

$$
p_{i \mid k}^{(t+1)}=\frac{\sum_{j=1}^{|y|} n\left(x_{i}, y_{j}\right) P\left(k \mid x_{i}, y_{j}, \Theta^{(t)}\right)}{\sum_{i=1}^{|X|} \sum_{j=1}^{|y|} n\left(x_{i}, y_{j}\right) P\left(k \mid x_{i}, y_{j}, \Theta^{(t)}\right)}
$$

Similarly, the next parameters $q_{j \mid k}{ }^{(t+1)}$ is:

$$
q_{j \mid k}^{(t+1)}=\frac{\sum_{i=1}^{|X|} n\left(x_{i}, y_{j}\right) P\left(k \mid x_{i}, y_{j}, \Theta^{(t)}\right)}{\sum_{i=1}^{|X|} \sum_{j=1}^{|y|} n\left(x_{i}, y_{j}\right) P\left(k \mid x_{i}, y_{j}, \Theta^{(t)}\right)}
$$

The two steps of GEM algorithm for SMM at some $t^{\text {th }}$ iteration are shown in table 2.1.

E-step:

The conditional probability $P\left(k \mid x_{i}, y_{j}, \Theta^{(t)}\right)$ is calculated based on current parameter $\Theta^{(t)}$ $=\left(\alpha_{k}^{(t)}, p_{i \mid k}{ }^{(t)}, q_{j \mid k}^{(t)}\right)^{T}$, according to equation 2.11 .

$$
P\left(k \mid x_{i}, y_{j}, \Theta^{(t)}\right)=\frac{\alpha_{k}^{(t)} p_{i \mid k}^{(t)} q_{j \mid k}^{(t)}}{\sum_{l=1}^{K} \alpha_{l}^{(t)} p_{i \mid l}^{(t)} q_{j \mid l}^{(t)}}
$$

M-step:

The next parameter $\Theta^{(t+1)}=\left(\alpha_{k}{ }^{(t+1)}, p_{i \mid k^{(t+1)}}, q_{j \mid k}{ }^{(t+1)}\right)^{T}$, which is a maximizer of $Q\left(\Theta \mid \Theta^{(t)}\right)$ with subject to $\Theta$, is calculated by equation 2.12, equation 2.13, and equation 2.14.

$$
\begin{aligned}
\alpha_{k}^{(t+1)} & =\frac{\sum_{i=1}^{|X|} \sum_{j=1}^{|y|} n\left(x_{i}, y_{j}\right) P\left(k \mid x_{i}, y_{j}, \Theta^{(t)}\right)}{\sum_{i=1}^{|X|} \sum_{j=1}^{|y|} n\left(x_{i}, y_{j}\right)} \\
p_{i \mid k}^{(t+1)} & =\frac{\sum_{j=1}^{|y|} n\left(x_{i}, y_{j}\right) P\left(k \mid x_{i}, y_{j}, \Theta^{(t)}\right)}{\sum_{i=1}^{|X|} \sum_{j=1}^{|y|} n\left(x_{i}, y_{j}\right) P\left(k \mid x_{i}, y_{j}, \Theta^{(t)}\right)} \\
q_{j \mid k}^{(t+1)} & =\frac{\sum_{i=1}^{|X|} n\left(x_{i}, y_{j}\right) P\left(k \mid x_{i}, y_{j}, \Theta^{(t)}\right)}{\sum_{i=1}^{|X|} \sum_{j=1}^{|y|} n\left(x_{i}, y_{j}\right) P\left(k \mid x_{i}, y_{j}, \Theta^{(t)}\right)}
\end{aligned}
$$

Table 2.1. E-step and M-step of GEM algorithm for SMM

GEM algorithm converges at some $t^{t \text { th }}$ iteration. At that time, $\Theta^{*}=\Theta^{(t+1)}=\Theta^{(t)}$ is the SMM itself. When SMM is applied into soft clustering, dyadic data is clustered according to blocks and each $\alpha_{k}$ is coverage ratio of cluster $k$ (aspect $k$ ). 
The mixture model of dyadic data is called asymmetric mixture model (AMM) if $\alpha_{k}$ (s) are only independent from $x_{i}$ or from $y_{j}$. Without loss of generality, given $\alpha_{k}$ (s) are only independent from $y_{j}$ (of course, it is dependent on $x_{i}$ ), AMM is defined as follows (Hofmann \& Puzicha, Statistical Models for Co-occurrence Data, 1998, p. 3):

$$
P\left(x_{i}, y_{j} \mid \Theta\right)=p_{i} q_{j \mid i}=p_{i} \sum_{k=1}^{K} \alpha_{k \mid i} q_{j \mid k}
$$

The $\alpha_{k \mid i}$ is the probability of aspect $k$ given $x_{i}$.

Where $p_{i}$ is the probability of $x_{i}$.

$$
\alpha_{k \mid i}=P\left(k \mid x_{i}\right)
$$

$$
p_{i}=P\left(x_{i}\right)
$$

The $q_{j \mid k}$ is the conditional probability of $y_{j}$ given aspect $k$. Suppose $y_{j}$ is dependent from $x_{i}$ given $k$, we have:

$$
q_{j \mid k}=P\left(y_{j} \mid x_{i}, k\right)=P\left(y_{j} \mid k\right)
$$

Note, $q_{j \mid i}$ is the conditional probability of $y_{j}$ given $x_{i}$, which is defined as follows:

The joint probability of $x_{i}, y_{j}$, and $k$ is:

$$
q_{j \mid i}=P\left(y_{j} \mid x_{i}\right)=\sum_{k=1}^{K} \alpha_{k \mid i} q_{j \mid k}
$$

$$
P\left(x_{i}, y_{j}, k\right)=P\left(x_{i}\right) P\left(y_{j}, k \mid x_{i}\right)=P\left(x_{i}\right) P\left(k \mid x_{i}\right) P\left(y_{j} \mid x_{i}, k\right)=p_{i} \alpha_{k \mid i} P\left(y_{j} \mid k\right)=p_{i} \alpha_{k \mid i} q_{j \mid k}
$$

The parameter of AMM is $\Theta=\left(\alpha_{k \mid i}, p_{i}, q_{j \mid k}\right)^{T}$ in which there are $K(|X|+|\mathcal{Y}|)+|X|$ partial parameters $\alpha_{k \mid i}, p_{i}$, and $q_{j \mid k}$. Note,

$$
\sum_{k=1}^{K} \alpha_{k \mid i}=1, \sum_{i=1}^{|X|} p_{i}=1, \sum_{j=1}^{|y|} q_{j \mid k}=1
$$

By applying GEM, given dyadic sample $\mathcal{S}$, at the $t^{\text {th }}$ iteration of GEM, given current parameter $\Theta^{(t)}=\left(\alpha_{k}^{(t)}, p_{i \mid k}{ }^{(t)}, q_{j \mid k}{ }^{(t)}\right)^{T}$, the conditional expectation $Q\left(\Theta \mid \Theta^{(t)}\right)$ is:

$$
\begin{aligned}
Q\left(\Theta \mid \Theta^{(t)}\right)= & \sum_{r=1}^{|\mathcal{S}|} \sum_{k=1}^{K} P\left(k \mid x_{i}(r), y_{j}(r), \Theta^{(t)}\right) \log \left(\alpha_{k \mid i} p_{i} q_{j \mid k}\right) \\
& =\sum_{i=1}^{|X|} \sum_{j=1}^{|y|} n\left(x_{i}, y_{j}\right) \sum_{k=1}^{K} P\left(k \mid x_{i}, y_{j}, \Theta^{(t)}\right)\left(\log \left(\alpha_{k \mid i}\right)+\log \left(p_{i}\right)\right. \\
& \left.+\log \left(q_{j \mid k}\right)\right)
\end{aligned}
$$

Where,

$$
P\left(k \mid x_{i}, y_{j}, \Theta^{(t)}\right)=\frac{\alpha_{k \mid i}^{(t)} p_{i}^{(t)} q_{j \mid k}^{(t)}}{\sum_{l=1}^{K} \alpha_{l \mid i}^{(t)} p_{i}^{(t)} q_{j \mid l}^{(t)}}
$$

Please refer to equation 1.4 to comprehend equation 2.17. Because there are three constraints

$$
\sum_{k=1}^{K} \alpha_{k \mid i}=1, \sum_{i=1}^{|X|} p_{i}=1, \sum_{j=1}^{|y|} q_{j \mid k}=1
$$

We use Lagrange duality method to maximize to maximize $Q\left(\Theta \mid \Theta^{(t)}\right)$. The Lagrange function la $\left(\Theta, \lambda \mid \Theta^{(t)}\right)$ is sum of $Q\left(\Theta \mid \Theta^{(t)}\right)$ and these constraints, as follows: 


$$
\begin{aligned}
l a\left(\Theta, \lambda \mid \Theta^{(t)}\right)= & Q\left(\Theta \mid \Theta^{(t)}\right)+\lambda_{1}\left(1-\sum_{k=1}^{K} \alpha_{k \mid i}\right)+\lambda_{2}\left(1-\sum_{i=1}^{|x|} p_{i}\right)+\lambda_{3}\left(1-\sum_{j=1}^{|y|} q_{j \mid k}\right) \\
& =\sum_{i=1}^{|X|} \sum_{j=1}^{|y|} n\left(x_{i}, y_{j}\right) \sum_{k=1}^{K} P\left(k \mid x_{i}, y_{j}, \Theta^{(t)}\right)\left(\log \left(\alpha_{k \mid i}\right)+\log \left(p_{i}\right)+\log \left(q_{j \mid k}\right)\right) \\
& +\lambda_{1}\left(1-\sum_{k=1}^{K} \alpha_{k \mid i}\right)+\lambda_{2}\left(1-\sum_{i=1}^{|x|} p_{i}\right)+\lambda_{3}\left(1-\sum_{j=1}^{|y|} q_{j \mid k}\right)
\end{aligned}
$$

Note, $\lambda=\left(\lambda_{1}, \lambda_{2}, \lambda_{3}\right)^{T}$ where $\lambda_{1} \geq 0, \lambda_{2} \geq 0$, and $\lambda_{3} \geq 0$ are called Lagrange multipliers. Of course, $l a\left(\Theta, \lambda \mid \Theta^{(t)}\right)$ is function of $\Theta$ and $\lambda$. The next parameters $\Theta^{(t+1)}$ that maximizes $Q\left(\Theta \mid \Theta^{(t)}\right)$ at Mstep of some $t^{\text {th }}$ iteration is solution of the equation formed by setting the first-order partial derivatives of Lagrange function regarding $\Theta$ and $\lambda$ to be zero.

The first-order partial derivative of Lagrange function regarding $\alpha_{k \mid i}$ is:

$$
\frac{\partial l a\left(\Theta, \lambda \mid \Theta^{(t)}\right)}{\partial \alpha_{k \mid i}}=\sum_{j=1}^{|y|} n\left(x_{i}, y_{j}\right) \frac{1}{\alpha_{k \mid i}} P\left(k \mid x_{i}, y_{j}, \Theta^{(t)}\right)-\lambda_{1}
$$

Setting this partial derivative to be zero, we obtain:

$$
\sum_{j=1}^{|y|} n\left(x_{i}, y_{j}\right) P\left(k \mid x_{i}, y_{j}, \Theta^{(t)}\right)-\alpha_{k \mid i} \lambda_{1}=0
$$

Summing the equation above over $K$ aspects $\{1,2, \ldots, K\}$, we have:

$\sum_{j=1}^{|y|} n\left(x_{i}, y_{j}\right) \sum_{k=1}^{K} P\left(k \mid x_{i}, y_{j}, \Theta^{(t)}\right)-\lambda_{1} \sum_{k=1}^{K} \alpha_{k}=0$

$\Leftrightarrow \sum_{j=1}^{|y|} n\left(x_{i}, y_{j}\right)-\lambda_{1}=0 \Leftrightarrow \lambda_{1}=\sum_{j=1}^{|y|} n\left(x_{i}, y_{j}\right)$

This means the next parameters $\alpha_{k \mid i}^{(t+1)}$ is:

$$
\alpha_{k \mid i}^{(t+1)}=\frac{\sum_{j=1}^{|y|} n\left(x_{i}, y_{j}\right) P\left(k \mid x_{i}, y_{j}, \Theta^{(t)}\right)}{\sum_{j=1}^{|y|} n\left(x_{i}, y_{j}\right)}
$$

The first-order partial derivative of Lagrange function regarding $p_{i}$ is:

$$
\frac{\partial l a\left(\Theta, \lambda \mid \Theta^{(t)}\right)}{\partial p_{i}}=\sum_{j=1}^{|y|} n\left(x_{i}, y_{j}\right) \frac{1}{p_{i}}-\lambda_{2}
$$

Setting this partial derivative to be zero, we obtain:

$$
\sum_{j=1}^{|y|} n\left(x_{i}, y_{j}\right)-p_{i} \lambda_{2}=0
$$

Summing the equation above over $\mathcal{X}$, we have:

$$
\begin{aligned}
& \sum_{i=1}^{|x|} \sum_{j=1}^{|y|} n\left(x_{i}, y_{j}\right)-\lambda_{2} \sum_{i=1}^{|x|} p_{i}=0 \\
& \Leftrightarrow \lambda_{2}=\sum_{i=1}^{|x|} \sum_{j=1}^{|y|} n\left(x_{i}, y_{j}\right)
\end{aligned}
$$


This means the next parameters $p_{i \mid k}^{(t+1)}$ is:

$$
p_{i}^{(t+1)}=\frac{\sum_{j=1}^{|y|} n\left(x_{i}, y_{j}\right)}{\sum_{i=1}^{|X|} \sum_{j=1}^{|y|} n\left(x_{i}, y_{j}\right)}
$$

The first-order partial derivative of Lagrange function regarding $q_{j \mid k}$ is:

$$
\frac{\partial l a\left(\Theta, \lambda \mid \Theta^{(t)}\right)}{\partial q_{j \mid k}}=\sum_{i=1}^{|X|} n\left(x_{i}, y_{j}\right) \frac{1}{q_{j \mid k}} P\left(k \mid x_{i}, y_{j}, \Theta^{(t)}\right)-\lambda_{3}
$$

Setting this partial derivative to be zero, we obtain:

$$
\sum_{i=1}^{|X|} n\left(x_{i}, y_{j}\right) P\left(k \mid x_{i}, y_{j}, \Theta^{(t)}\right)-q_{j \mid k} \lambda_{3}=0
$$

Summing the equation above over $\mathcal{Y}$, we have:

$\sum_{i=1}^{|x|} \sum_{j=1}^{|y|} n\left(x_{i}, y_{j}\right) P\left(k \mid x_{i}, y_{j}, \Theta^{(t)}\right)-\lambda_{3} \sum_{j=1}^{|y|} q_{j \mid k}$

$\Leftrightarrow \sum_{i=1}^{|X|} \sum_{j=1}^{|y|} n\left(x_{i}, y_{j}\right) P\left(k \mid x_{i}, y_{j}, \Theta^{(t)}\right)-\lambda_{3} \Leftrightarrow \lambda_{3}=\sum_{i=1}^{|X|} \sum_{j=1}^{|y|} n\left(x_{i}, y_{j}\right) P\left(k \mid x_{i}, y_{j}, \Theta^{(t)}\right)$

This means the next parameters $q_{j \mid k}^{(t+1)}$ is:

$$
q_{j \mid k}^{(t+1)}=\frac{\sum_{i=1}^{|X|} n\left(x_{i}, y_{j}\right) P\left(k \mid x_{i}, y_{j}, \Theta^{(t)}\right)}{\sum_{i=1}^{|X|} \sum_{j=1}^{|\mathcal{Y}|} n\left(x_{i}, y_{j}\right) P\left(k \mid x_{i}, y_{j}, \Theta^{(t)}\right)}
$$

The two steps of GEM algorithm for AMM at some $t^{\text {th }}$ iteration are shown in table 2.2.

E-step:

The conditional probability $P\left(k \mid x_{i}, y_{j}, \Theta^{(t)}\right)$ is calculated based on current parameter $\Theta^{(t)}$ $=\left(\alpha_{k \mid i}{ }^{(t)}, p_{i}^{(t)}, q_{j \mid k^{(t)}}\right)^{T}$, according to equation 2.17 .

M-step:

$$
P\left(k \mid x_{i}, y_{j}, \Theta^{(t)}\right)=\frac{\alpha_{k \mid i}^{(t)} p_{i}^{(t)} q_{j \mid k}^{(t)}}{\sum_{l=1}^{K} \alpha_{l \mid i}^{(t)} p_{i}^{(t)} q_{j \mid l}^{(t)}}
$$

The next parameter $\Theta^{(t+1)}=\left(\alpha_{k \mid i}{ }^{(t+1)}, p_{i}{ }^{(t+1)}, q_{j \mid k}{ }^{(t+1)}\right)^{T}$, which is a maximizer of $Q\left(\Theta \mid \Theta^{(t)}\right)$ with subject to $\Theta$, is calculated by equation 2.18 , equation 2.19 , and equation 2.20 .

$$
\begin{aligned}
\alpha_{k \mid i}^{(t+1)} & =\frac{\sum_{j=1}^{|y|} n\left(x_{i}, y_{j}\right) P\left(k \mid x_{i}, y_{j}, \Theta^{(t)}\right)}{\sum_{j=1}^{|y|} n\left(x_{i}, y_{j}\right)} \\
p_{i}^{(t+1)} & =\frac{\sum_{j=1}^{|y|} n\left(x_{i}, y_{j}\right)}{\sum_{i=1}^{|X|} \sum_{j=1}^{|y|} n\left(x_{i}, y_{j}\right)} \\
q_{j \mid k}^{(t+1)} & =\frac{\sum_{i=1}^{|X|} n\left(x_{i}, y_{j}\right) P\left(k \mid x_{i}, y_{j}, \Theta^{(t)}\right)}{\sum_{i=1}^{|x|} \sum_{j=1}^{|y|} n\left(x_{i}, y_{j}\right) P\left(k \mid x_{i}, y_{j}, \Theta^{(t)}\right)}
\end{aligned}
$$

Table 2.2. E-step and M-step of GEM algorithm for AMM

GEM algorithm converges at some $t^{\text {th }}$ iteration. At that time, $\Theta^{*}=\Theta^{(t+1)}=\Theta^{(t)}$ is the AMM itself. When AMM is applied into soft clustering, dyadic data is clustered vertically (horizontally) and each $\alpha_{k \mid i}$ is coverage ratio of cluster $k$ (aspect $k$ ) according to $x_{i}$. Soft clustering with AMM is also called one-side clustering. 
Product-space mixture model (PMM) is derived from SMM with a minor change that the aspect set $\{1,2, \ldots, K\}$ is Cartesian product of $\mathcal{X}$-aspect set $\left\{1,2, \ldots, K_{\mathcal{X}}\right\}$ and $\mathcal{Y}$-aspect set $\{1$, $\left.2, \ldots, K_{y}\right\}$. In other words, the aspect space is still symmetric but is checked (stripped) according to two directions $\mathcal{X}$ and $\mathcal{Y}$.

$$
\begin{aligned}
& \{1,2, \ldots, K\}=\left\{1,2, \ldots, K_{X}\right\} \times\left\{1,2, \ldots, K_{\mathcal{Y}}\right\} \\
& K=K_{X} K_{y}
\end{aligned}
$$

For every $k$ belongs to $\{1,2, \ldots, K\}$, there always exists a respective pair: $k_{\chi} \in\left\{1,2, \ldots, K_{\chi}\right\}$ and $k_{y} \in\left\{1,2, \ldots, K_{y}\right\}$. However, for each $k_{x}$ or each $k_{y}$, there are many respective $k$.

$$
\begin{aligned}
& k \sim\left\{k_{x}, k_{y}\right\} \\
& k_{x} \sim \text { many } k \\
& k_{y} \sim \text { many } k
\end{aligned}
$$

The sign " $\sim$ " denotes correspondence. PMM is defined as follows (Hofmann \& Puzicha, Statistical Models for Co-occurrence Data, 1998, p. 4):

$$
P\left(x_{i}, y_{j} \mid \Theta\right)=\sum_{k=1}^{K} \alpha_{k} p_{i \mid k_{x}} q_{j \mid k_{y}}
$$

As usual, $\alpha_{k}$ is the probability of aspect $c_{k}$ but $p_{i \mid k_{X}}$ is the probability of $x_{i}$ given $k_{X}$ of $k$ and $q_{j \mid k_{y}}$ is the probability of $y_{j}$ given $k_{y}$ of $k$.

The joint probability of $x_{i}, y_{j}$, and $k$ is:

$$
\begin{aligned}
& p_{i \mid k x}=P\left(x_{i} \mid k_{x}\right) \\
& q_{j \mid k_{y}}=P\left(y_{j} \mid k_{y}\right)
\end{aligned}
$$

$$
\begin{aligned}
P\left(x_{i}, y_{j}, k\right)= & P(k) P\left(x_{i}, y_{j} \mid k\right)=\alpha_{k} P\left(x_{i} \mid k\right) P\left(y_{j} \mid k\right)=\alpha_{k} P\left(x_{i} \mid k_{x}\right) P\left(y_{j} \mid k_{y}\right) \\
& =\alpha_{k} p_{i \mid k_{x}} q_{j \mid k y}
\end{aligned}
$$

The parameter of PMM is $\Theta=\left(\alpha_{k}, p_{i \mid k_{x}}, q_{j \mid k_{y}}\right)^{T}$ in which there are $K+K_{X}|\mathcal{X}|+K_{y}|\mathcal{Y}|$ partial parameters $\alpha_{k}, p_{i \mid k x}$, and $q_{j \mid k_{y}}$. Note,

$$
\sum_{k=1}^{K} \alpha_{k}=1, \sum_{i=1}^{|x|} p_{i \mid k x}=1, \sum_{j=1}^{|y|} q_{j \mid k_{y}}=1
$$

Learning PMM is like learning SMM and so it is not necessary to duplicate the expansion of $Q\left(\Theta \mid \Theta^{(t)}\right)$. The two steps of GEM algorithm for PMM at some $t^{\text {th }}$ iteration are shown in table 2.3 .

E-step:

The conditional probabilities $P\left(k \mid x_{i}, y_{j}, \Theta^{(t)}\right), P\left(k_{x} \mid x_{i}, y_{j}, \Theta^{(t)}\right)$, and $P\left(k_{y} \mid x_{i}, y_{j}, \Theta^{(t)}\right)$ are calculated based on current parameter $\Theta^{(t)}=\left(\alpha_{k}^{(t)}, p_{i \mid k x}^{(t)}, q_{j \mid k y}^{(t)}\right)^{T}$, according to equation 2.24, equation 2.25, and equation 2.26 .

$$
\begin{gathered}
P\left(k \mid x_{i}, y_{j}, \Theta^{(t)}\right)=\frac{\alpha_{k}^{(t)} p_{i \mid k_{x}}^{(t)} q_{j \mid k_{y}}^{(t)}}{\sum_{l=1}^{K} \alpha_{l}^{(t)} p_{i \mid l_{x}}^{(t)} q_{j \mid l_{y}}^{(t)}} \\
P\left(k_{x} \mid x_{i}, y_{j}, \Theta^{(t)}\right)=\sum_{k: k_{x} \sim k} P\left(k \mid x_{i}, y_{j}, \Theta^{(t)}\right) \\
P\left(k_{y} \mid x_{i}, y_{j}, \Theta^{(t)}\right)=\sum_{k: k_{y} \sim k} P\left(k \mid x_{i}, y_{j}, \Theta^{(t)}\right)
\end{gathered}
$$

Please refer to equation 1.4 to comprehend equation 2.24 . M-step: 
The next parameter $\Theta^{(t+1)}=\left(\alpha_{k}^{(t+1)}, p_{i \mid k x}^{(t+1)}, q_{j \mid k y}^{(t+1)}\right)^{T}$, which is the maximizer of $Q(\Theta \mid$ $\Theta^{(t)}$ ) with subject to $\Theta$, is calculated by equation 2.27 , equation 2.28 , and equation 2.29 .

$$
\begin{aligned}
\alpha_{k}^{(t+1)} & =\frac{\sum_{i=1}^{|X|} \sum_{j=1}^{|y|} n\left(x_{i}, y_{j}\right) P\left(k \mid x_{i}, y_{j}, \Theta^{(t)}\right)}{\sum_{i=1}^{|X|} \sum_{j=1}^{|y|} n\left(x_{i}, y_{j}\right)} \\
p_{i \mid k x}^{(t+1)} & =\frac{\sum_{j=1}^{|y|} n\left(x_{i}, y_{j}\right) P\left(k_{x} \mid x_{i}, y_{j}, \Theta^{(t)}\right)}{\sum_{i=1}^{|X|} \sum_{j=1}^{|y|} n\left(x_{i}, y_{j}\right) P\left(k_{x} \mid x_{i}, y_{j}, \Theta^{(t)}\right)} \\
q_{j \mid k y}^{(t+1)} & =\frac{\sum_{i=1}^{|X|} n\left(x_{i}, y_{j}\right) P\left(k_{y} \mid x_{i}, y_{j}, \Theta^{(t)}\right)}{\sum_{i=1}^{|X|} \sum_{j=1}^{|y|} n\left(x_{i}, y_{j}\right) P\left(k_{y} \mid x_{i}, y_{j}, \Theta^{(t)}\right)}
\end{aligned}
$$

Table 2.3. E-step and M-step of GEM algorithm for PMM

GEM algorithm converges at some $t^{\text {th }}$ iteration. At that time, $\Theta^{*}=\Theta^{(t+1)}=\Theta^{(t)}$ is the PMM itself. When PMM is applied into soft clustering, dyadic data is clustered in checked (stripped) and each $\alpha_{k}$ is coverage ratio of cluster $k$ (aspect $k$ ) but such cluster $k$ corresponds to a pair of cluster $k_{x}$ and cluster $k_{y}$. Soft clustering with PMM is also called two-side clustering.

\section{Predicting unaccomplished co-occurrent values}

This section is the main subject of this research in which some extensions of dyadic mixture models are used to predict unaccomplished values in valued dyadic data. When $\mathcal{S}$ is valued dyadic data in which every co-occurrence $\left(x_{i}, y_{j}\right)$ is associated with value $z$ from random variable $Z$ then, SMM is reformed as follows:

AMM is reformed as follows:

$$
f\left(x_{i}, y_{j}, Z \mid \Theta\right)=\sum_{k=1}^{K} \alpha_{k} p_{i \mid k} q_{j \mid k} f_{k}\left(Z \mid \varphi_{k}\right)
$$

$$
f\left(x_{i}, y_{j}, Z \mid \Theta\right)=p_{i} \sum_{k=1}^{K} \alpha_{k \mid i} q_{j \mid k} f_{k}\left(Z \mid \varphi_{k}\right)
$$

$\mathrm{PMM}$ is reformed as follows:

$$
f\left(x_{i}, y_{j}, Z \mid \Theta\right)=\sum_{k=1}^{K} \alpha_{k} p_{i \mid k_{x}} q_{j \mid k y} f_{k}\left(Z \mid \varphi_{k}\right)
$$

Where $f_{k}\left(Z \mid \varphi_{k}\right)$ is the $k^{\text {th }}$ PDF of $Z$ corresponding to the aspect $k$, in which $\varphi_{k}$ is parameter of $f_{k}\left(Z \mid \varphi_{k}\right)$. Of course, the parameter $\Theta$ now must include all $\varphi_{k}$. It is possible to consider that

Moreover, $Z$ is only dependent on $k$.

$$
f_{k}\left(Z \mid \varphi_{k}\right)=f\left(Z \mid k, \varphi_{k}\right)
$$

$$
f\left(Z \mid x_{i}, k, \varphi_{k}\right)=f\left(Z \mid k, \varphi_{k}\right)=f_{k}\left(Z \mid \varphi_{k}\right)
$$

Note, suppose $x_{i}$ and $y_{j}$ (as well as $y_{j}$ given $x_{i}$ ) are independent from $Z$ given aspect $k$, which is the hint to reform these models.

$$
\begin{aligned}
& P\left(x_{i}, y_{j} \mid k, Z\right)=P\left(x_{i}, y_{j} \mid k\right) \\
& P\left(y_{j} \mid x_{i}, Z, k\right)=P\left(y_{j} \mid x_{i}, k\right)
\end{aligned}
$$

For example, within SMM, the joint PDF of $x_{i}, y_{j}, Z$, and $k$ is:

$$
\begin{aligned}
f\left(x_{i}, y_{j}, Z, k\right) & =P(k) P\left(x_{i}, y_{j}, Z \mid k\right)=\alpha_{k} P\left(x_{i}, y_{j} \mid k, Z\right) f\left(Z \mid k, \varphi_{k}\right)=\alpha_{k} P\left(x_{i}, y_{j} \mid k\right) f_{k}\left(Z \mid \varphi_{k}\right) \\
& =\alpha_{k} P\left(x_{i} \mid k\right) P\left(y_{j} \mid k\right) f_{k}\left(Z \mid \varphi_{k}\right)=\alpha_{k} p_{i \mid k} q_{j \mid k} f_{k}\left(Z \mid \varphi_{k}\right)
\end{aligned}
$$

Within AMM, the joint PDF of $x_{i}, y_{j}, Z$, and $k$ is: 


$$
\begin{aligned}
f\left(x_{i}, y_{j}, Z, k\right) & =P\left(x_{i}\right) P\left(y_{j}, Z, k \mid x_{i}\right)=p_{i} P\left(k \mid x_{i}\right) P\left(y_{j}, Z \mid x_{i}, k\right) \\
& =p_{i} \alpha_{k \mid i} P\left(y_{j} \mid x_{i}, Z, k\right) f\left(Z \mid x_{i}, k, \varphi_{k}\right)=p_{i} \alpha_{k \mid i} P\left(y_{j} \mid x_{i}, k\right) f\left(Z \mid k, \varphi_{k}\right) \\
& =p_{i} \alpha_{k \mid i} P\left(y_{j} \mid k\right) f_{k}\left(Z \mid \varphi_{k}\right)=p_{i} \alpha_{k \mid i} q_{j \mid k} f_{k}\left(Z \mid \varphi_{k}\right)
\end{aligned}
$$

Within PMM, the joint PDF of $x_{i}, y_{j}, Z$, and $k$ is:

$$
\begin{aligned}
f\left(x_{i}, y_{j}, Z, k\right) & =P(k) P\left(x_{i}, y_{j}, Z \mid k\right)=\alpha_{k} P\left(x_{i}, y_{j} \mid Z, k\right) f\left(Z \mid k, \varphi_{k}\right)=\alpha_{k} P\left(x_{i}, y_{j} \mid k\right) f_{k}\left(Z \mid \varphi_{k}\right) \\
& =\alpha_{k} P\left(x_{i} \mid k_{x}\right) P\left(y_{j} \mid k_{y}\right) f_{k}\left(Z \mid \varphi_{k}\right)=\alpha_{k} p_{i \mid k x} q_{j \mid k_{y}} f_{k}\left(Z \mid \varphi_{k}\right)
\end{aligned}
$$

It is only necessary to estimate $\varphi_{k}$ because how to estimate other partial parameters was mentioned in section 2. By reforming the conditional expectation $Q\left(\Theta \mid \Theta^{(t)}\right)$, it is easy to find out that the next parameter $\varphi_{k}{ }^{(t+1)}$ is solution of following equation:

$$
\sum_{r=1}^{|\mathcal{S}|} P\left(k \mid x_{i}(r), y_{j}(r), \Theta^{(t)}\right) \frac{\mathrm{d} \log \left(f_{k}\left(Z(r) \mid \varphi_{k}\right)\right)}{\mathrm{d} \varphi_{k}}
$$

Where $P\left(k \mid x_{i}(r), y_{j}(r), \Theta^{(t)}\right)$ is specified by equation 2.11, equation 2.17, and equation 2.24 for $\mathrm{SMM}, \mathrm{AMM}$, and PMM, respectively. Especially, if $f_{k}\left(Z \mid \varphi_{k}\right)$ distributed normally, the next parameter $\varphi_{k}{ }^{(t+1)}=\left(\mu_{k}{ }^{(t+1)}, \Sigma_{k}{ }^{(t+1)}\right)^{T}$ containing mean $\mu_{k}{ }^{(t+1)}$ and covariance matrix $\Sigma_{k}{ }^{(t+1)}$ is calculated as follows:

$$
\begin{aligned}
\mu_{k}^{(t+1)} & =\frac{\sum_{r=1}^{|\mathcal{S}|} P\left(k \mid x_{i}(r), y_{j}(r), \Theta^{(t)}\right) Z(r)}{\sum_{i=1}^{|\mathcal{S}|} P\left(k \mid x_{i}(r), y_{j}(r), \Theta^{(t)}\right)} \\
\Sigma_{k}^{(t+1)} & =\frac{\sum_{r=1}^{|\mathcal{S}|} P\left(k \mid x_{i}(r), y_{j}(r), \Theta^{(t)}\right)\left(\left(Z(r)-\mu_{k}^{(t+1)}\right)\left(Z(r)-\mu_{k}^{(t+1)}\right)^{T}\right)}{\sum_{r=1}^{|\mathcal{S}|} P\left(k \mid x_{i}(r), y_{j}(r), \Theta^{(t)}\right)}
\end{aligned}
$$

Where $P\left(k \mid x_{i}(r), y_{j}(r), \Theta^{(t)}\right)$ is specified by equation 2.11 , equation 2.17 , and equation 2.24 for SMM, AMM, and PMM, respectively. Please refer to (Nguyen, 2020, pp. 83-84) to comprehend equation 3.5.

In valued dyadic sample $\mathcal{S}$, many co-occurrences $\left(x_{i}, y_{j}\right)$ are not existent and thus, it is required to predict or estimate $Z$ value of inexistent co-occurrence $\left(x_{i}, y_{j}\right)$. This $Z$ value is called unaccomplished co-occurrent value or unaccomplished associative value. A so-called expected co-occurrent (EC) method is used to estimate $Z$. Firstly, it is necessary to define the conditional PDF of $Z$ given $x_{i}$ and $y_{j}$. According to Bayes' rule, we have:

$$
f\left(Z \mid x_{i}, y_{j}, \Theta\right)=\frac{f\left(x_{i}, y_{j}, Z\right)}{\int_{Z} f\left(x_{i}, y_{j}, Z \mid \Theta\right) \mathrm{d} Z}=\frac{f\left(x_{i}, y_{j}, Z \mid \Theta\right)}{f\left(x_{i}, y_{j} \mid \Theta\right)}
$$

Then, $Z$ value of inexistent co-occurrence $\left(x_{i}, y_{j}\right)$ is estimated by an estimate $\hat{Z}$ which is the expectation of $Z$ given the conditional $\operatorname{PDF} f\left(Z \mid x_{i}, y_{j}, \Theta\right)$, as follows:

$$
\hat{Z}=E(Z \mid \Theta)=\int_{Z} Z f\left(Z \mid x_{i}, y_{j}, \Theta\right) \mathrm{d} Z
$$

In short, EC method is specified by equation 3.6 and equation 3.7. Now we expend the two equations for SMM, AMM, and PMM. The conditional PDF $f\left(Z \mid x_{i}, y_{j}, \Theta\right)$ of SMM is:

$$
f\left(Z \mid x_{i}, y_{j}, \Theta\right)=\frac{\sum_{k=1}^{K} \alpha_{k} p_{i \mid k} q_{j \mid k} f_{k}\left(Z \mid \varphi_{k}\right)}{\sum_{k=1}^{K} \alpha_{k} p_{i \mid k} q_{j \mid k}}
$$

Following is the proof of equation 3.8.

$$
\begin{aligned}
f\left(Z \mid x_{i}, y_{j}, \Theta\right) & =\frac{\sum_{k=1}^{K} \alpha_{k} p_{i \mid k} q_{j \mid k} f_{k}\left(Z \mid \varphi_{k}\right)}{\int_{Z} \sum_{k=1}^{K} \alpha_{k} p_{i \mid k} q_{j \mid k} f_{k}\left(Z \mid \varphi_{k}\right)}=\frac{\sum_{k=1}^{K} \alpha_{k} p_{i \mid k} q_{j \mid k} f_{k}\left(Z \mid \varphi_{k}\right)}{\sum_{k=1}^{K} \alpha_{k} p_{i \mid k} q_{j \mid k} \int_{Z} f_{k}\left(Z \mid \varphi_{k}\right)} \\
& =\frac{\sum_{k=1}^{K} \alpha_{k} p_{i \mid k} q_{j \mid k} f_{k}\left(Z \mid \varphi_{k}\right)}{\sum_{k=1}^{K} \alpha_{k} p_{i \mid k} q_{j \mid k}}
\end{aligned}
$$

Similarly, the conditional PDF $f\left(Z \mid x_{i}, y_{j}, \Theta\right)$ of AMM is: 


$$
f\left(Z \mid x_{i}, y_{j}, \Theta\right)=\frac{\sum_{k=1}^{K} \alpha_{k \mid i} q_{j \mid k} f_{k}\left(Z \mid \varphi_{k}\right)}{\sum_{k=1}^{K} \alpha_{k \mid i} q_{j \mid k}}
$$

The conditional PDF $f\left(Z \mid x_{i}, y_{j}, \Theta\right)$ of PMM is:

$$
f\left(Z \mid x_{i}, y_{j}, \Theta\right)=\frac{\sum_{k=1}^{K} \alpha_{k} p_{i \mid k_{x}} q_{j \mid k_{y}} f_{k}\left(Z \mid \varphi_{k}\right)}{\sum_{k=1}^{K} \alpha_{k} p_{i \mid k_{x}} q_{j \mid k_{y}}}
$$

Obviously, equation 3.8, equation 3.9, and equation 3.10 are extensions of equation 3.6.

The estimate $\hat{Z}$ for SMM is:

The estimate $\hat{Z}$ for AMM is:

$$
\hat{Z}=\frac{\sum_{k=1}^{K} \alpha_{k} p_{i \mid k} q_{j \mid k} E_{k}\left(Z \mid \varphi_{k}\right)}{\sum_{k=1}^{K} \alpha_{k} p_{i \mid k} q_{j \mid k}}
$$

The estimate $\hat{Z}$ for PMM is:

$$
\hat{Z}=\frac{\sum_{k=1}^{K} \alpha_{k \mid i} q_{j \mid k} E_{k}\left(Z \mid \varphi_{k}\right)}{\sum_{k=1}^{K} \alpha_{k \mid i} q_{j \mid k}}
$$

$$
\hat{Z}=\frac{\sum_{k=1}^{K} \alpha_{k} p_{i \mid k_{x}} q_{j \mid k_{y}} E_{k}\left(Z \mid \varphi_{k}\right)}{\sum_{k=1}^{K} \alpha_{k} p_{i \mid k_{x}} q_{j \mid k_{y}}}
$$

Where $E_{k}\left(Z \mid \varphi_{k}\right)$ is expectation of $Z$ given the $k^{\text {th }} \operatorname{PDF}$ of $Z$ :

$$
E_{k}\left(Z \mid \varphi_{k}\right)=\int_{Z} Z f_{k}\left(Z \mid \varphi_{k}\right) \mathrm{d} Z
$$

If $f_{k}\left(Z \mid \varphi_{k}\right)$ is multinormal PDF with mean $\mu_{k}$ and covariance matrix $\Sigma_{k}$ then, we have $E_{k}\left(Z \mid \varphi_{k}\right)=$ $\mu_{k}$. Note, equation 3.11, equation 3.12, and equation 3.13 are extensions of equation 3.7.

Hofmann's research (Hofmann, Latent Semantic Models for Collaborative Filtering, 2004) is different from EC method when Hofmann assumed that $f_{k}\left(Z \mid \varphi_{k}\right)$ is dependent on both $k$ and $x_{i}$ so that $f_{k}\left(Z \mid \varphi_{k}\right)$ is replaced by $f_{i k}\left(Z \mid \varphi_{i k}\right)$.

$$
f_{i k}\left(Z \mid \varphi_{i k}\right)=f\left(Z \mid x_{i}, k, \varphi_{i k}\right)=f\left(Z \mid x_{i}, y_{j}, k, \varphi_{i k}\right)
$$

Hofmann also assumed that (Hofmann \& Puzieha, Latent Class Models for Collaborative Filtering, 1999, p. 690)

$$
P\left(k \mid x_{i}, y_{j}\right)=P\left(k \mid y_{j}\right)=\frac{P(k) P\left(y_{j} \mid k\right)}{\sum_{k=1}^{K} P(k) P\left(y_{j} \mid k\right)}=\frac{\alpha_{k} q_{j \mid k}}{\sum_{k=1}^{K} \alpha_{k} q_{j \mid k}} \propto \alpha_{k} q_{j \mid k}
$$

The sign " $\propto$ " indicates the proportion. Therefore, according to Hofmann, the conditional PDF $f\left(Z \mid x_{i}, y_{j}, \Theta\right)$ was defined as follows:

$$
f\left(Z \mid x_{i}, y_{j}, \Theta\right)=\sum_{k=1}^{K} P\left(k \mid x_{i}, y_{j}\right) f\left(Z \mid x_{i}, y_{j}, k, \varphi_{i k}\right) \propto \sum_{k=1}^{K} \alpha_{k} q_{j \mid k} f_{i k}\left(Z \mid \varphi_{i k}\right)
$$

The estimate $\hat{Z}$ is still calculated by equation 3.7 except that $f\left(Z \mid x_{i}, y_{j}, \Theta\right)$ was defined by equation 3.15. As a result, equation 3.15 is the real mixture model of Hofmann in (Hofmann, Latent Semantic Models for Collaborative Filtering, 2004) and then Hofmann applied EM algorithm to learn parameters $\alpha_{k}, q_{j \mid k}$, and $\varphi_{i k}$. Therefore, Hofmann's mixture model in (Hofmann, Latent Semantic Models for Collaborative Filtering, 2004) is not mixture models of co-occurrences $\left(x_{i}, y_{j}\right)$ specified by equation 2.9 (SMM), equation 2.15 (AMM), and 2.23 (PMM). Hofmann's mixture model is appropriate to collaborative filtering.

\section{Conclusions}

Essentially, learning dyadic data with models such as SMM, AMM, and PMM is unsupervised learning and it is easy to apply these models into soft clustering. Predicting or estimating unaccomplished values is essential to make a weighted sum of centroids over all clusters. Currently, an unaccomplished value is estimated based on pre-knowledge of an existent pair of 
two objects ( $\mathcal{X}$-object and $\mathcal{Y}$-object). As a result, an estimate $\hat{Z}$ is fixed if the two objects are fixed. In future, I try to find out another method to take advantages of more than two existent objects with a set of values. Combination of dyadic mixture model and regression model is a candidate method but how to prove and explain it is still fuzzy problem.

\section{References}

Hofmann, T. (2004, January). Latent Semantic Models for Collaborative Filtering. $A C M$ Transactions on Information Systems (TOIS), 22(1), 89-115. doi:10.1145/963770.963774

Hofmann, T., \& Puzicha, J. (1998). Statistical Models for Co-occurrence Data. Massachusetts Institute of Technology, Artificial Intelligence Laboratory. MIT Publisher. Retrieved from https://dspace.mit.edu/bitstream/handle/1721.1/7253/AIM-1625.pdf?sequence=2

Hofmann, T., \& Puzieha, J. (1999). Latent Class Models for Collaborative Filtering. In T. Dean (Ed.), Proceedings of the Sixteenth International Joint Conference on Artificial Intelligence (IJCAI '99) (pp. 688-693). San Francisco, CA, USA: Morgan Kaufmann. Retrieved from https://dl.acm.org/citation.cfm?id=687583

Hofmann, T., Puzicha, J., \& Jordan, M. I. (1998). Learning from Dyadic Data. In M. J. Kearns, S. A. Solla, \& D. A. Cohn (Ed.), Advances in Neural Information Processing Systems 11 (NIPS 1998). 11, pp. 466-472. Denver: MIT Press. Retrieved from https://papers.nips.cc/paper/1503-learning-from-dyadic-data

Nguyen, L. (2020). Tutorial on EM algorithm. MDPI. Preprints. doi:10.20944/preprints201802.0131.v8 